\title{
A new protocol for concomitant needle aspiration biopsy and localization of solitary pulmonary nodules
}

Young Jo Sa, Jae Jun Kim, Young Du Kim, Sung Bo Sim and Seok Whan Moon*

\begin{abstract}
Background: Pulmonary nodules may require thoracoscopic resection in cases where percutaneous needle aspiration (PCNA) is non-diagnostic or not technically feasible. We developed a new protocol to localize pulmonary nodules concomitantly with PCNA. We retrospectively reviewed the use of concomitant PCNA and preoperative localization under computed tomography (CT) guidance.

Methods: From Jan 2006 to Dec 2013, we performed PCNA and localization concomitantly on 34 pulmonary nodules (in 33 patients) using self-made, platinum microcoils. Patients in which PCNA results were less likely to be non-diagnostic and who were anticipating thoracoscopy were eligible to participate in this study. The CT-guided PCNA biopsy and microcoil localization was performed on the day of the VATS in the CT suite. The PCNA specimen was sent to the pathologist for frozen section pathology. If diagnosis of the lesion was not confirmed by PCNA or was primary lung cancer, the patient was moved to the operating room for VATS surgery.

Results: Between Jan 2006 and Dec 2013, concomitant PCNA and localization were successfully performed on 34 pulmonary nodules from 33 patients (one patient had two nodules). Of the 34 nodules, seven were diagnosed pathologically using PCNA, and 27 nodules that could not be diagnosed by PCNA were excised by thoracoscopic resection without additional procedures or time because of concomitant localization. There were no deaths or significant morbidities. Minor complications included three incidents of lung hemorrhage and five of pneumothorax (two required closed thoracostomy drainage). Of 34 nodules in which both PCNA and localization were used, thoracoscopic resections were performed on 33, lobectomies were performed concomitantly with thoracoscopic resection on 11. Intraoperative fluoroscopy was used to detect 33 of 34 nodules localized using the platinum microcoil $(97.06 \%)$ or to guide stapling during thoracoscopic resection.
\end{abstract}

Conclusions: The advantages of this technique are 1) there is no need for further localization during thoracoscopy even in cases of unsuccessful PCNA, 2) it is more effective with respect to both cost and time, and 3) it provides greater patient comfort.

Keywords: Pulmonary nodule, Preoperative localization, Thoracoscopy, PCNA

\section{Background}

With widespread clinical use of high-resolution computed tomography (CT), numerous small pulmonary nodules are frequently detected during lung cancer screenings, follow-up examinations after cancer treatment, and during routine examinations for chronic

\footnotetext{
*Correspondence: swmoon@catholic.ac.kr

Department of Thoracic and Cardiovascular Surgery, College of Medicine,

The Catholic University of Korea, 222 Banpo-daero, Seocho-gu, Seoul 137-701, Republic of Korea
}

diseases [1]. Approximately 150,000 new cases a year are diagnosed in the United States and approximately $40 \sim 50 \%$ of these are malignant [2]. Percutaneous needle aspiration (PCNA) biopsy and transbronchial needle biopsy are well-established useful procedures but are less reliable to rule out malignancy than video-assisted thoracoscopic surgery (VATS) because of inadequate tissue samples or biopsy failure [3]. VATS is a useful minimally invasive tool but cannot always diagnose small, deep seated sub-pleural nodules, which are 
frequently neither visible nor palpable [4]. We devised a new protocol that will yield a definitive diagnosis from a needle aspiration biopsy and will also localize the nodule, which is very helpful for thoracoscopic pulmonary nodule resection when PCNA is non-diagnostic.

We introduce here our protocol for preoperative concomitant PCNA and solitary pulmonary nodule (SPN) localization using our microcoil and we review the pertinent literature.

\section{Methods}

\section{Patients}

From Jan 2006 to Dec 2013, we performed PCNA and localization concomitantly on 34 pulmonary nodules (in 33 patients) using self-made, fragmented (5 $\mathrm{mm}$ in length, $0.5 \mathrm{~mm}$ in diameter), platinum microcoils (Easimarker, Tae Woong Medical Co., Korea, NO. 0195053). Patients in which PCNA results were less likely to be non-diagnostic and who were anticipating thoracoscopy were eligible to participate in this study. We have performed preoperative CT-guided localization in VATS resection if the nodule meets one or more criteria: (1) maximum diameter of nodule $5 \mathrm{~mm}$ or less, (2) a distance from the outer margin of the nodule to the nearest pleural surface $>5 \mathrm{~mm}$, (3) low-density on CT. With the cooperation of the pulmonologist and radiologist, the plan was to perform PCNA and localization concomitantly. Patients with severe emphysematous lungs, central lesions, and lesions attached to bronchi or pulmonary vessels were excluded.

The Institutional Review Board of Yeouido St. Mary's Hospital, The Catholic University of Korea approved retrospective data retrieval (approval No.SC15RISE0024). All patients agreed to undergo the procedure after being informed of the risks and benefits as well as the alternatives for management.

\section{CT-guided PCNA Biopsy and Microcoil Localization}

The CT-guided PCNA biopsy and microcoil localization was performed on the day of the VATS in the CT suite. A radiologist performed the procedure under local anesthesia. The patient was placed in a supine or prone position depending on the location of the lesion. The target lesion was detected on $1 \sim 3 \mathrm{~mm}$ thick axial CT sections taken on full inspiration, then punctured with a 21-gauge Chiba needle (Calibrated Chiba Stylet needle, Manan Medical Products Inc. Ill) into or just near the nodule. Once the needle tip was identified to be within the nodule or just in contact with it, the stylet was removed from the needle. Localization was performed followed by PCNA biopsy. For localization, the microcoil was completely loaded in the needle, and then introduced into the nodule or just near the nodule by pushing down the stylet. Additional CT images were routinely obtained to assess the position of the microcoil relative to the lesion and the pleural surface, and to identify any complications. If the localization failed or was inadequate, the procedure was repeated. In some cases, PCNA was performed before localization (Fig. 1). The biopsied specimen of PCNA was sent to the pathologist for frozen section pathology. If diagnosis of the lesion was primary lung cancer or not confirmed by PCNA, the patient was moved to the operating room for VATS surgery.

\section{Fluoroscopy-Assisted Thoracoscopic Surgery (FATS)}

Thoracoscopic surgery was performed only when PCNA results were not obtained or an additional specimen was needed. Thoracoscopic surgery was performed under single lung ventilation using a double lumen endotracheal tube with three thoracoscopic ports that were made for a $5-\mathrm{mm}$ or $10-\mathrm{mm}$ thoracoscope, a grasper, and an endo-stapler. During thoracoscopic surgery, the fluoroscopic unit was used to detect the microcoil within or around the nodule, and to identify a generous margin for the thoracoscopic resection. Most of the needle puncture sites on the visceral pleura were identified on the thoracoscopic view, and the inserted parenchymal microcoils were clearly visualized fluoroscopically. The resected lung containing the microcoil was withdrawn into an endoscopic retrieval bag, and confirmed fluoroscopically (Fig. 1). The specimen was sent to the pathologist for frozen section pathology. If the lesion was determined to be a benign or completely resected metastatic nodule, the operation was finished. If the lesion was a non-small cell lung cancer that was surgically resectable, a lobectomy with mediastinal lymph node dissection was performed.

\section{Results}

The study included 24 men and 9 women. The average age was $57.06 \pm 10.40$ (range 36 to 77 ) years. The diagnosis was not confirmed pathologically in all lesions, but 10 nodules (in nine patients) were suspected to be pulmonary metastases from previous malignancies. Previous malignancies included uterine cervical cancer $(n=1)$, and hepatocellular $(n=2)$, colorectal $(n=4)$ and musculoskeletal $(n=2)$ cancers. Of 34 pulmonary nodules, a pathologic diagnosis was made in seven using CT-guided PCNA to obtain the specimen. Twenty-six patients were moved from the surgery room and 27 intrapulmonary nodules (two nodules in one patient) were successfully wedge-resected and pathologies confirmed on frozen section without additional time-consuming procedures. The diameter of the nodules ranged from 3 to $34 \mathrm{~mm}$ with a mean $14.37 \pm 7.88 \mathrm{~mm}$. The distance from the outer margin of the nodule to the nearest pleural surface ranged from 3 to $31 \mathrm{~mm}$ with a mean of $12.15 \pm 7.44 \mathrm{~mm}$ (Table 1). There were no deaths and no significant 


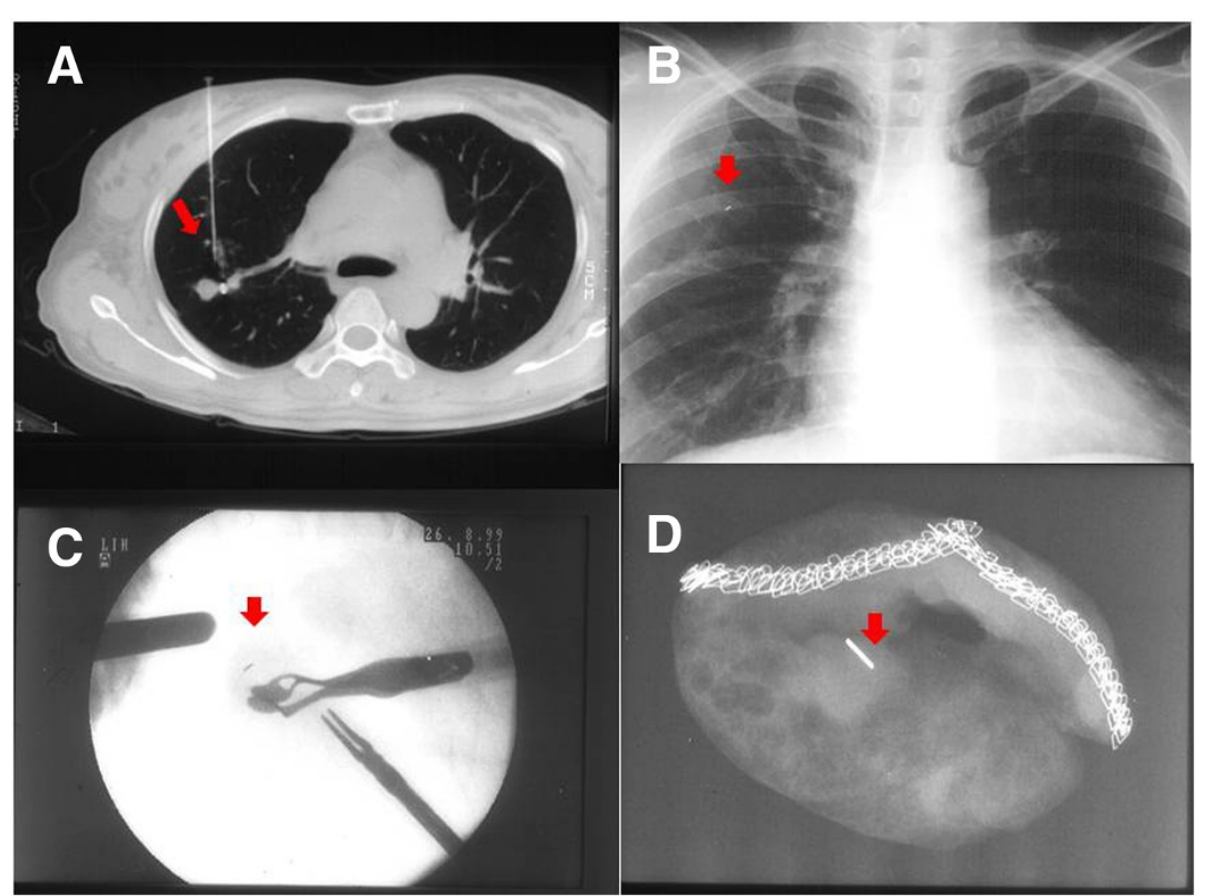

Fig. 1 a Once the needle tip is confirmed to be just in contact with the nodule, the stylet is removed from the needle, and a microcoil (arrow) is inserted by pushing the stylet using CT guidance. After localization, PCNA (percutaneous needle aspiration) is simultaneously performed to obtain specimens for cytologic examination. $\mathbf{b}$ This chest x-ray shows the radiopacity (arrow) created by a microcoil inserted during PCNA. c During thoracoscopy, the microcoil (arrow) in contact with the nodule is clearly visualized on the monitor of portable fluoroscopic unit, which helps to detect the nodule and plan the stapling resection. $\mathbf{d}$ The wedge resection of the pulmonary nodule, including the microcoil (arrow), is identified using fluoroscopy

morbidities. Minor complications related to the PCNA and localization procedure included three incidents of lung hemorrhages and five of pneumothorax (two required mini-closed thoracostomy drainage). There was one microcoil-detection failure due to intrathoracic displacement of the microcoil during FATS. The success rate of our localization technique was $97.06 \%$ (33/34). In one localization-failed patient, we easily performed thoracoscopic wedge resection.

Fluoroscopy assisted wedge resections were successfully performed on all 27 nodules. Histopathologic diagnoses (including diagnosis by PCNA) were successfully obtained in 34 nodules (diagnostic accuracy, $100 \%$ ) and included 11 primary cancers, nine metastatic lung cancers, nine granulomas, four tuberculous granulomas and one hamartoma (Fig. 2). All 11 patients with primary lung cancer underwent lobectomy with mediastinal lymph node dissection.

\section{Discussion}

Improved imaging techniques such as high resolution, single breath, or spiral CT scanning, result in more frequent identification of small and often sub-centimetric SPNs [4]. Although most SPNs are benign [5, 6], the pathology of the nodule is extremely important in a patient who has previously had cancer even if the nodule is small and peripheral. In addition, even in small pulmonary lesions less than $1 \mathrm{~cm}$, the overall malignancy rate is as high as, or slightly lower than that in nodules larger than $1 \mathrm{~cm}$ [7-9].

A PCNA biopsy is a well-established, useful procedure. However, the diagnostic accuracy of PCNA depends on the size and location of the lesion, as well as the guidance technique, and decreases from over $90 \%$ to $25 \%$ when the malignant nodule is small (less than $1 \mathrm{~cm}$ ), and to $70 \%$ when the lesion is benign $[9,10]$. As many as $29 \%$ of patients whose conditions were not diagnosed as malignant on trans-thoracic needle biopsy were ultimately found to have carcinoma [11]. Therefore, VATS has become a useful excisional biopsy tool for the diagnosis of indeterminate pulmonary nodules. Most VATS excisional biopsies are performed after obtaining a definitive diagnosis using PCNA has failed. VATS and localization are performed as separate procedures on separate days. To reduce the discomfort, cost and time of repeating localization and VATS, we have developed a simultaneous protocol that combines PCNA biopsy with localization for VATS for use in cases PCNA results were less likely to be non-diagnostic and who were anticipating thoracoscopy. 
Table 1 Patient characteristics and pulmonary nodule pathology results

\begin{tabular}{|c|c|c|c|c|c|c|c|c|}
\hline Patient & Sex & $\begin{array}{l}\text { Age } \\
\text { (year) }\end{array}$ & $\begin{array}{l}\text { Diameter of } \\
\text { nodule }(\mathrm{mm})\end{array}$ & $\begin{array}{l}\text { Depth from } \\
\text { pleura }(\mathrm{mm})\end{array}$ & $\begin{array}{l}\text { Histologic } \\
\text { confirmation }\end{array}$ & Localization & Final pathology & Definite treatment \\
\hline 1 & M & 53 & 10 & 11 & VATS & Success & Primary Lung cancer & $\begin{array}{l}\text { Lobectomy with mediastinal } \\
\text { lymph node dissection }\end{array}$ \\
\hline 2 & M & 57 & 27 & 5 & PCNA & Success & Tuberculous granuloma & None \\
\hline 3 & M & 56 & 31 & 13 & PCNA & Success & Primary Lung cancer & $\begin{array}{l}\text { Lobectomy with mediastinal } \\
\text { lymph node dissection }\end{array}$ \\
\hline 4 & $\mathrm{~F}$ & 56 & 12 & 7 & VATS & Success & Tuberculous granuloma & VATS \\
\hline 5 & M & 63 & 11 & 9 & VATS & Success & $\begin{array}{l}\text { Metastasis from Hepatocellular } \\
\text { carcinoma }\end{array}$ & VATS \\
\hline 6 & M & 66 & 17 & 5 & VATS & Success & Tuberculous granuloma & VATS \\
\hline 7 & $\mathrm{~F}$ & 36 & 6 & 7 & VATS & Success & Metastasis from Uterine cervix cancer & VATS \\
\hline 8 & M & 57 & 27 & 5 & PCNA & Success & Primary Lung cancer & $\begin{array}{l}\text { Lobectomy with mediastinal } \\
\text { lymph node dissection }\end{array}$ \\
\hline 9 & $\mathrm{~F}$ & 53 & 10 & 11 & VATS & Success & Hamartoma & VATS \\
\hline 10 & $\mathrm{~F}$ & 66 & 34 & 22 & PCNA & Success & Primary Lung cancer & $\begin{array}{l}\text { Lobectomy with mediastinal } \\
\text { lymph node dissection }\end{array}$ \\
\hline 11 & M & 77 & 15 & 7 & VATS & Success & Small cell Lung carcinoma & VATS \\
\hline 12 & M & 48 & 21 & 11 & VATS & Success & Carcinoid tumor & VATS \\
\hline 13 & M & 53 & 21 & 19 & VATS & Success & Granuloma & VATS \\
\hline 14 & M & 68 & 15 & 30 & PCNA & Success & Primary Lung cancer & $\begin{array}{l}\text { Lobectomy with mediastinal } \\
\text { lymph node dissection }\end{array}$ \\
\hline 15 & M & 44 & 22 & 25 & VATS & Success & $\begin{array}{l}\text { Metastasis from Hepatocellular } \\
\text { carcinoma }\end{array}$ & VATS \\
\hline 16 & M & 52 & 11 & 9 & VATS & Success & Granuloma & VATS \\
\hline 17 & M & 49 & 13 & 16 & VATS & Success & Tuberculous granuloma & VATS \\
\hline 18 & M & 39 & 20 & 7 & VATS & Success & Metastasis from Musculoskeletal cancer & VATS \\
\hline 19 & $\mathrm{~F}$ & 43 & 3 & 4 & VATS & Success & Metastasis from Musculoskeletal cancer & VATS \\
\hline 20 & M & 53 & 10 & 3 & VATS & Failure & Granuloma & VATS \\
\hline 21 & M & 62 & 21 & 23 & PCNA & Success & Primary Lung cancer & $\begin{array}{l}\text { Lobectomy with mediastinal } \\
\text { lymph node dissection }\end{array}$ \\
\hline 22 & M & 52 & 13 & 9 & VATS & Success & Granuloma & VATS \\
\hline 23 & M & 74 & 9 & 11 & VATS & Success & Primary Lung cancer & VATS \\
\hline 24 & M & 67 & 22 & 9 & PCNA & Success & Primary Lung cancer & $\begin{array}{l}\text { Lobectomy with mediastinal } \\
\text { lymph node dissection }\end{array}$ \\
\hline 25 & M & 67 & 7 & 5 & VATS & Success & Granuloma & VATS \\
\hline 26 & M & 75 & 11 & 12 & VATS & Success & Primary Lung cancer & $\begin{array}{l}\text { Lobectomy with mediastinal } \\
\text { lymph node dissection }\end{array}$ \\
\hline 27 & $\mathrm{~F}$ & 51 & 7 & 9 & VATS & Success & Granuloma & VATS \\
\hline 28 & $\mathrm{~F}$ & 44 & 6 & 14 & VATS & Success & Metastasis from Colorectal cancer & VATS \\
\hline 29 & $\mathrm{~F}$ & 69 & 11 & 22 & VATS & Success & Granuloma & VATS \\
\hline 30 & M & 67 & 5 & 31 & VATS & Success & Metastasis from Colorectal cancer & VATS \\
\hline 31 & M & 61 & 11 & 9 & VATS & Success & Metastasis from Colorectal cancer & VATS \\
\hline 32 & M & 54 & 6 & 7 & VATS & Success & $\begin{array}{l}\text { Metastasis from Colorectal cancer/ } \\
\text { Granuloma }\end{array}$ & VATS \\
\hline 33 & M & 51 & 9 & 14 & VATS & Success & Granuloma & VATS \\
\hline
\end{tabular}

To localize small or deeply-seated difficult lesions preoperatively or intraoperatively, several techniques have been devised such as pleural marking with dyes [12] and cyanoacrylate adhesive [13], a hook-wire system [14, 15], fluoroscopy with contrast media such as barium sulfate or lipiodol [16-18], microcoils [19] or bronchoscopy- 


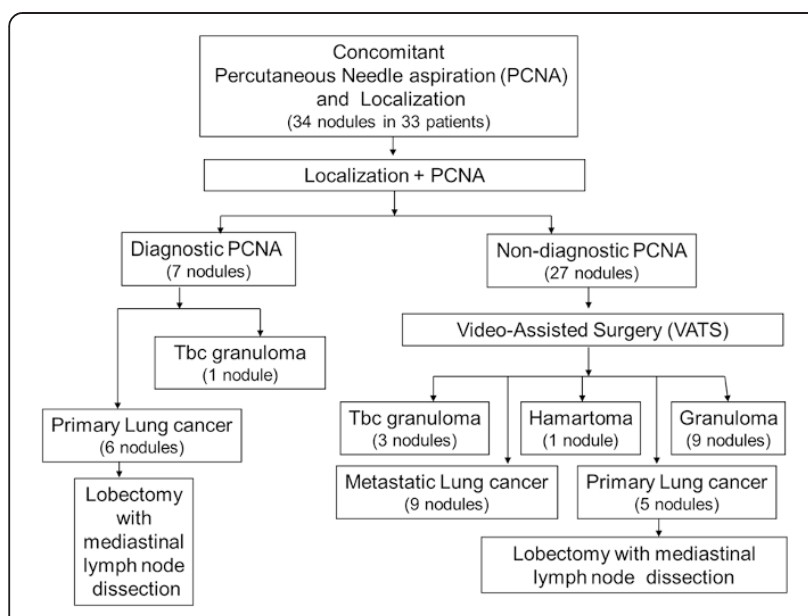

Fig. 2 Summary of 34 nodules from 33 patients using concomitant percutaneous needle aspiration and localization protocol

guided transbronchial metallic coils [20], and endothoracic [21] and radio-guided ultrasonography [22, 23]. Because our localization was performed with PCNA preoperatively, we had to consider pre-operative localization using a contrast media such as barium sulfate or lipiodol, hook-wire systems, and microcoils. Even if the localization material is left permanently, which can also happen when the pathologic diagnosis is obtained by PCNA, localization will not result in any problems. Contrast media injected into or around the pulmonary nodule can cause chronic inflammation in the lung (unpublished data; we observed chronic localized inflammation in our animal study). The hook-wire system also has a removal problem when the diagnosis is confirmed using PCNA. Therefore, of these methods, we selected a radiopaque wire segment, platinum microcoil, as our localization method.

In our previous study [24], we reported the results of using fluoroscopy-assisted thoracoscopy to resect small intrapulmonary lesions after preoperative computed tomography guided localization using fragmented platinum microcoils. The platinum microcoil localization was easily manipulated, did not influence the dimension or pathologic status of the nodule, and was free of a specific time frame of use before the operation. Platinum microcoils have been used as radiomarkers in stereotactic radiation treatment planning for brain tumors, head and neck tumors, uterine cervical cancer, and in marking the border or center of tumors before primary chemotherapy for breast cancer $[25,26]$. The procedure can be considered so inert that no adverse side effects should be expected, even if the coils are left permanently.

With this concomitant PCNA and localization protocol, we successfully performed fluoroscopy-assisted thoracoscopic wedge resections on 27 nodules. We obtained pathologic diagnoses in all 27 nodules without another procedure although additional time $(\leq 5 \mathrm{~min})$ for localization with PCNA was necessary. This concomitant localization technique was successful in 33 of 34 nodules $(97.06 \%)$. We consider our concomitant PCNA and localization protocol to be a successful, time-saving, and efficient method for accurate diagnosis and resection of indeterminate solitary nodules.

We have performed preoperative localization if the nodule meets one or more criteria, which are (1) maximum diameter of the nodule $<5 \mathrm{~mm}$, (2) a distance from the outer margin of the nodule to the nearest pleural surface $>5 \mathrm{~mm}$, (3) low-density imaging on CT. Regarding localization criteria discussed in the literature, Saito et al. [27] demonstrated that a linear discriminant analysis using both size and depth factors (depth = $0.836 \times$ size -2.811 ) might serve as an indication for preoperative localization of nodules using VATS resection. Suzuki et al. [28] considered a preoperative marking as absolutely indicated when the distance is $>10 \mathrm{~mm}$. According to Saito et al. criteria, $62.11 \%(22 / 36)$ of the pulmonary nodules in our cases required localization; only $44.44 \%(16 / 36)$ of our pulmonary nodules met the criteria of Suzuki et al. Further studies are needed to establish conditions requiring VATS, and possible conditions to localize concomitantly with PCNA to diagnose pulmonary nodules in particular. Although additional studies are needed, we think that our technique saves the merits of PCNA and VATS procedure harmoniously for diagnosing and treating the pulmonary nodules. Because there will be no need for another thoracoscopic CT localization, the cost-benefits of using this procedure can be demonstrated in labor savings, reduced CT scanning and film costs, decreased use of the localizing needle, and shorter hospital stays for patients.

\section{Conclusions}

We conclude that concomitant PCNA and localization is the preferred technique to establish a definite diagnosis when PCNA results were less likely to be non-diagnostic and in who were anticipating thoracoscopy.

\section{Completing interests}

The authors declare that they have no competing interests.

\section{Authors' contributions}

YJS: having been involved in drafting the manuscript or revising it, analysis and interpretation of data. JJK, YDK, SBS: acquisition of data. SWM: acquisition of data, analysis and interpretation of data, final approval of the version to be published. All authors read and approved the final manuscript.

\section{Acknowledgements}

Name: 24th World Congress of the World Society of Cardiothoracic surgeons Location: Geneva, Switzerland

Date: 2014.09.06 to 2014.09.10

Received: 24 April 2015 Accepted: 23 July 2015

Published online: 29 July 2015 


\section{References}

1. Tsuchiya R. Investigation and management of nodules less than one centimeter in size. In: Shields TW, LoCicero III J, Reed CE, Feins RH, editors. General Thoracic Surgery. 7th ed. Philadelphia, PA: Lippincott Williams \& Wilkins; 2009. p. 1307-10.

2. Lillington GA, Caskey $\mathrm{Cl}$. Evaluation and management of solitary and multiple pulmonary nodules. Clin Chest Med. 1993;14:111-9.

3. Alzahouri K, Velten M, Arveux P, Woronoff-Lemsi MC, Jolly D, Guillemin F. Management of SPN in France. Pathways for definitive diagnosis of solitary pulmonary nodule: a multicentre study in 18 French districts. BMC Cancer. 2008;8:93-101.

4. Chen S, Zhou J, Zhang J, Hu H, Luo X, Zhang Y, et al. Video-assisted thoracoscopic solitary pulmonary nodule resection after CT-guided hookwire localization: 43 cases report and literature review. Eur J Cardiothorac Surg. 2007;32(6):843-7.

5. Leef 3rd JL, Klein IS. The solitary pulmonary nodule. Radiol Clin North Am. 2002;40:123-43.

6. Yankelvits DF, Hensche Cl. Lung cancer: small solitary puplmonary nodules. Radiol Clin North Am. 2000;38:1-9.

7. Munden RF, Pugatch RD, Liptay MJ, Sugarbaker DJ, Le LU. Small pulmonary lesions detected at CT: clinical importance. Radiology. 1997;202:105-10.

8. Ginsberg MS, Griff SK, Go BD, Yoo HH, Schwart LH, Panicek DM. Pulmonary nodules resected at video-assisted thoracoscopic surgery: etiology in 426 patients. Radiology. 1999;213:277-82.

9. Mitruka S, Landreneau RJ, Mack MJ, Fetterman LS, Gammie J, Bartley S, et al. Diagnosing the indeterminate pulmonary nodule: Percutaneous biopsy versus thoracoscopy. Surgery. 1995;118(4):676-84.

10. Layfield LJ, Coogan A, Johnston WW, Patz EF. Transthoracic fine needle aspiration biopsy. Sensitivity in relation to guidance technique and lesion size and location. Acta Cytol. 1996;40(4):687-90.

11. Calhoun P, Feldman PS, Armstrong P, Black WC, Pope TL, Minor GR, et al. The clinical outcome of needle aspiration of the lung when cancer is not diagnosed. Ann Thorac Surg. 1986;41(6):592-6.

12. Wicky S, Mayor B, Schnyder P. Methylene blue localizations of pulmonary nodules under $C$-guidance: a new procedure used before thoracoscopic resections. Int Surg. 1997;82(1):15-7.

13. Yoshida J, Nagai K, Nishimura M, Takahashi K. Computed tomographyfluoroscopy guided injection of cyanoacrylate to mark a pulmonary nodule for thoracoscopic resection. Jpn J Thorac Cardiovasc Surg. 1999;47(5):210-3.

14. Pittet O, Christodoulou M, Pezzetta E, Schmidt S, Schnyder P, Ris HB. Videoassisted thoracoscopic resection of a small pulmonary nodule after computed tomography-guided localization with a hook-wire system. Experience in 45 consecutive patients. World J Surg. 2007;31(3):575-8.

15. Ciriaco P, Negri G, Puglisi A, Nicoletti R, Del Maschio A, Zannini P. Videoassisted thoracoscopic surgery for pulmonary nodules: rationale for preoperative computed tomography-guided hookwire localization. Eur J Cardiothorac Surg. 2004;25(3):429-33.

16. Moon SW, Wang YP, Jo KH, Kwack MS, Kim SW, Kwon OK, et al. Fluoroscopy-aided thoracoscopic resection of pulmonary nodule localized with contrast media. Ann Thorac Surg. 1999;68(5):1815-20.

17. Watanabe K, Nomori H, Ohtsuka T, Kaji M, Naruke T, Suemasu K. Usefulness and complications of computed tomography-guided lipiodol marking for fluoroscopy-assisted thoracoscopic resection of small pulmonary nodules: experience with 174 nodules. J Thorac Cardiovasc Surg. 2006;132(2):320-4.

18. Kawanaka K, Nomori H, Mori T, Ikeda K, Ikeda O, Tomiguchi S, et al. Marking of small pulmonary nodules before thoracoscopic resection: injection of lipiodol under CT-fluoroscopic guidance. Acad Radiol. 2009;16(1):39-45.

19. Powell Tl, Jangra D, Clifton JC, Lara-Guerra H, Church N, English J, et al. Peripheral lung nodules: fluoroscopically guided video-assisted thoracoscopic resection after computed tomography-guided localization using platinum microcoils. Ann Surg. 2004;240(3):481-9.

20. Miyoshi T, Kondo K, Takizawa H, Kenzaki K, Fujino H, Sakiyama S, et al. Fluoroscopy-assisted thoracoscopic resection of pulmonary nodules after computed tomography-guided bronchoscopic metallic coil marking. J Thorac Cardiovasc Surg. 2006;131(3):704-10.

21. Matsumoto S, Hirata T, Ogawa E, Fukuse T, Ueda H, Koyama T, et al. Ultrasonographic evaluation of small nodules in the peripheral lung during video-assisted thoracic surgery (VATS). Eur J Cardiothorac Surg. 2004;26(3):469-73
22. Sortini D, Feo C, Maravegias K, Carcoforo P, Pozza E, Liboni A, et al. Intrathoracoscopic localization techniques. Review of literature. Surg Endosc. 2006;20(9):1341-7.

23. Chella A, Lucchi M, Ambrogi MC, Menconi G, Melfi FM, Gonflotti A, et al. A pilot study of the role of TC-99 radionuclide in localization of pulmonary nodular lesions for thoracoscopic resection. Eur J Cardiothorac Surg. 2000;18(1):17-21

24. Moon SW, Cho DG, Cho KD, Kang CU, Jo MS, Park HJ. Fluoroscopy-Assisted thoracoscopic resection for small intrapulmonary lesions after preoperative computed tomography-guided localization using fragmented platinum microcoils. Thorac Cardiovasc Surg. 2012;60(6):413-8.

25. Hay KD, Hindley A, Sharp I. A simple percutaneous inserter for radiopaque gold seeds used in radiotherapy treatment planning. Australas Radiol. 1998;42:169-71.

26. Reynolds HE, Lesnefsky MH, Jackson VP. Tumor marking before primary chemotherapy for breast cancer. AJR. 1999;173:919-20.

27. Saito H, Minamiya Y, Matsuzaki I, Tozawa K, Taguchi K, Nakagawa T, et al. Indication for preoperative localization of small peripheral pulmonary nodules in thoracoscopic surgery. J Thorac Cardiovasc Surg. 2002;124(6):1198-202.

28. Suzuki K, Nagai K, Yoshida J, Ohmatsu H, Takahashi K, Nishimura M, et al Video-assisted thoracoscopic surgery for small indeterminate pulmonary nodules. Chest. 1999;115:563-8.

\section{Submit your next manuscript to BioMed Central and take full advantage of:}

- Convenient online submission

- Thorough peer review

- No space constraints or color figure charges

- Immediate publication on acceptance

- Inclusion in PubMed, CAS, Scopus and Google Scholar

- Research which is freely available for redistribution 